\title{
Research on the Development Technology of Cross Platform Hybrid Mobile Application Based on HTML5
}

\author{
Qiao Yan ${ }^{1, *}$, Guyu $\mathrm{Hu}^{1}$, Guiqiang $\mathrm{Ni}^{1}$, Jinsong Jiang ${ }^{1}$ and Junxian Long ${ }^{2}$ \\ ${ }^{1}$ PLA University of Science and Technology, Nanjing 210007, China \\ ${ }^{2}$ Unit 69066 of PLA, Urumqi 830001, China \\ ${ }^{*}$ Corresponding author
}

\begin{abstract}
Because there are many differences in the different operating systems of intelligent equipment, application software adaptation workload will be multiplied. Based on HTML5 cross platform development framework, such as PhoneGap, Sencha Touch, Titanium and AppCan and so on, can easily solve the cross platform problem, reduce software development cycle and the costs of software development and maintenance. This paper mainly introduced PhoneGap, Sencha Touch, Titanium and AppCan cross platform development framework, compared to their advantages and disadvantages, and analyzed the advantages and disadvantages of cross platform mobile hybrid mobile development mode based on HTML5.Finally, this paper looked forward to the development of HTML5 and development technology of cross platform hybrid mobile application based on HTML5.
\end{abstract}

Keywords-cross platform mobile development framework; HTML5 hybrid application; PhoneGap; Sencha Touch; Titanium; AppCan

\section{INTRODUCTION}

With the development of $4 \mathrm{G}$ network technology and mobile Internet, the popularity of mobile intelligent terminal is becoming higher and higher. The latest report which was released by Global well-known research firm Gartner shows that the global smart phone sales reached 1 billion 500 million in 2016, to 2020 the overall mobile phone market is expected to reach 1 billion 900 million [1]. Gartner released the first quarter of 2016 global smartphone market research report shows that in the smart phone operating system market, Android accounted for $84.1 \%$, iOS accounted for $14.8 \%$, Windows Phone, blackberry and other market share are less than $1 \%$ [2], the detailed data are shown in Table I.

TABLE I. 2016 MAJOR OPERATING SYSTEMS IN THE FIRST QUARTER OF THE GLOBAL SMART PHONE MARKET SHARE

\begin{tabular}{|l|l|}
\hline \multicolumn{1}{|c|}{ Operating System } & \multicolumn{1}{c|}{ Market Share(\%) } \\
\hline Android & 84.1 \\
\hline iOS & 14.8 \\
\hline Windows Phone & 0.7 \\
\hline BlackBerry & 0.2 \\
\hline Others & 0.2 \\
\hline
\end{tabular}

Each platform has its own development language and software development kit, and it can't be compatible between different platforms [3]. In order to get more users, the application has to be published for the different versions of different platforms' terminals. However, the application development of different platforms need to learn new language and environment, which means spending more time and money, and the latter maintenance must be separated which also increases the cost of software development. Therefore, how to make a simple and rapid cross platform adaptation has become a problem to be solved in the mobile applications development. Table II lists the programming languages required for the application development of the mainstream mobile operating system.

TABLE II. THE DEVELOPMENT AND APPLICATION OF THE MAJOR SMART PHONE OPERATING SYSTEM PROGRAMMING LANGUAGE

\begin{tabular}{|l|l|}
\hline \multicolumn{1}{|c|}{ Operating System } & \multicolumn{1}{c|}{ Programming Language } \\
\hline Android & Java \\
\hline iOS & Objective-C \\
\hline Windows Phone & C\#,.Net \\
\hline BlackBerry & Java \\
\hline
\end{tabular}

\section{Classification OF Mobile ApPliCAtion}

Mainstream applications on mobile devices can be divided into three types: native application (Native App), hybrid application (Hybrid App) and web application (Web App). Native App use a native language in the mobile operating system, such as Java for Android, Objective-C for iOS, to carry out the application code, it is run directly in the mobile operating system, thus the performance and user experience is best, but development work is difficulty and cost is high; Web App is websites viewed on a mobile device in a mobile browser, designed specifically to fit a mobile device screen size, work well on a mobile device and are accessed through a mobile browser, which has a strong dependence on the browser, the user experience is poor, but the development difficulty and cost is low; Hybrid App run a web application through a separate browser kernel WebView which is a native application, so it needn't depend on the system browser, performance and user experience is good, the development difficulty and cost is low.

The fundamental difference among the three main applications lies in the different design architecture. Figure I shows the comparison of the design architecture of the three applications, and also describes how they access the database or WebService to load the data [4]. 
Among the three applications, only Hybrid App and Web App can cross platform, but access to Web App only in the browser, can't be called mobile application in the traditional sense, Hybrid App can retain native application user experience as far as possible to cross platform.

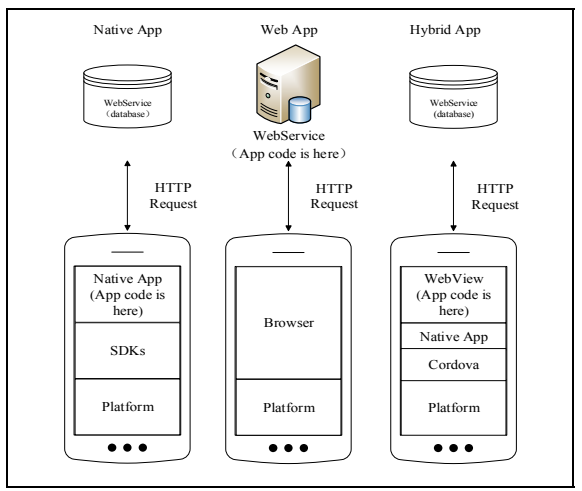

FIGURE I. COMPARISON OF ARCHITECTURES FOR NATIVE APP, WEB APP, AND HYBRID APP

With the development of HTML5, HTML5 cross platform characteristics allow hybrid application development advantages to further expand. Compared with the traditional native application development, cross platform hybrid mobile application development technology based on HTML5 is known for its cross platform, low cost and easy development, it is favored by many developers.

\section{CROSS PlatForm DEVELOPMENT FramWORK AND ITS COMPARISON}

Because of the adaptation of different mobile operating systems, equipment types, a mobile application development often need to spend a lot of time, cross platform mobile development framework free developers from heavy adaptation work. Cross platform mobile application development framework based on HTML5 allows developers to use HTML, CSS and JavaScript to quickly build a cross platform mobile hybrid applications, shorten the development cycle, saving development costs.

\section{A. PhoneGap and Cordova}

PhoneGap [5] is an open source, free cross platform mobile development framework based on HTML, CSS and JavaScript, which was launched by Adobe in 2011. The framework provides an application layer adapter which adapted the underlying native API to a web application development interface, developers can use HTML, CSS and JavaScript and other web front-end development technology to build the application's user interface layer and business logic layer, call the PhoneGap API interface to interact with the operating system underlying, to implement geographical location, gravity sensing, file system access, camera calls, contacts and so on. PhoneGap not only provides mobile phone based local system API, but also provides a wealth of plug-in support, developers can also write their own plug-ins to meet the development needs.

At present, PhoneGap support six mobile platform, Apple's IOS, Google's Android, RIM's BlackBerry and Microsoft's
Windows Phone, Windows and Ubuntu, basically can cover $90 \%$ of the mobile market for mobile devices. Therefore, PhoneGap can basically achieve "developed once, run everywhere", in the cross platform adaptation, developers only need to use re packaged, avoid the secondary development, greatly improve the efficiency of development. Because PhoneGap follows the W3C standard, web developers can become directly application developers.

PhoneGap is already a very successful and mature mobile cross platform solutions, it has abundant third-party resources and mature industry chain: developers can choose Ionic, jQuery mobile and Sencha Touch JavaScript libraries to accelerate development progress, using AppMobi and Tiggr integrated development environment to develop and debug (by dragging a layout, line coding and run a mobile device simulator), can also choose PhoneGap Bulid(a professional online compiler tools), removed from the various compiler environment problems.

Cordova [6] is Apache's open source project which is donated by Adobe's PhoneGap, is the core of PhoneGap code, and is also core engine to drive PhoneGap. And Adobe will continue to use the combination of Cordova, PhoneGap Build and Shadow Adobe to provide PhoneGap. So the core of PhoneGap is Cordova, the main difference between PhoneGap and Cordova is command line tools and PhoneGap building services. The architecture of Cordova is shown in Figure II:

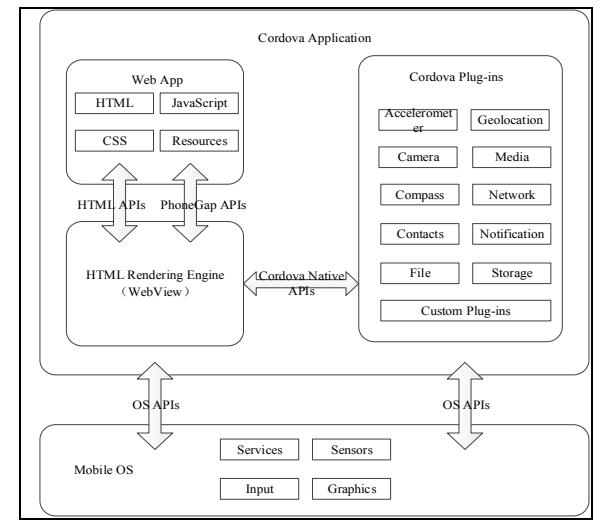

FIGURE II. THE ARCHITECTURE OF CORDOVA

Cordova provides a set of device related API, through this group of API, mobile applications can access the native device functions via JavaScript, such as cameras, microphones, and so on. Currently, the latest version of Cordova is 6.x, support for the following mobile operating systems: iOS, Android, Ubuntu, Blackberry, Windows Phone. Compared to PhoneGap, Cordova is widely used, has more detailed documentation, more stable performance.

\section{B. Sencha Touch}

Sencha Touch [7] is the leading MVC-based JavaScript framework for building cross-platform mobile web applications Sencha Touch is the world's first application framework built specifically to leverage HTML5, CSS3, and JavaScript for the highest level of power, flexibility, and optimization. Sencha Touch leverages hardware acceleration techniques to provide high-performance UI components for mobile devices. Sencha 
Touch supports Android, iOS, Windows Phone, Microsoft Surface Pro and RT, and BlackBerry devices.

Sencha Touch was born out of the culture and many of the ideas from the venerable Ext JS framework, and provide a simple but powerful app architecture that leverages the Model View Controller (MVC) pattern. Sencha Touch solves cross-platform mobile app development problems by giving developers the tools necessary to build cross-platform applications that mimic natively compiled applications, while making full use of HTML5 and CSS3.

Sencha Touch has the following characteristics:

- High-Performance, Native-Looking UI Widgets.

- Adaptive Layouts, Animations, and Smooth Scrolling.

- Backend Agnostic Data Package.

- Advanced Mobile Charting Package

- Device Features and Native Packaging.

\section{Appcelerator Titanium}

Titanium [8] application program uses HTML, JavaScript and CSS to develop, supports PHP, Ruby and Python, based on Titanium platform, users can develop application program fast and deploy it expediently, and these application program can use local APIs to implement many functions and characteristics that common Web application was unable to complete. Titanium allows application developers to write a variety of smart phone mobile operating system applications, such as iOS, Android, Windows Phone, BlackBerry and so on.

\begin{tabular}{|c|c|c|c|c|c|}
\hline \multicolumn{3}{|c|}{ Your Application } & \multicolumn{3}{|c|}{ Your Application } \\
\hline UI API & Phone API & Optional Modules & UI API & \begin{tabular}{|l||} 
Phone API \\
\end{tabular} & Optional Modules \\
\hline \multicolumn{3}{|c|}{ JavaScript-Objective C Bridge } & \multicolumn{3}{|c|}{ JavaScript-Java Bridge } \\
\hline \multicolumn{3}{|c|}{ iPhone OS } & \multicolumn{3}{|c|}{ Android OS } \\
\hline \multicolumn{3}{|c|}{ Native PPhone APP } & \multicolumn{3}{|c|}{ Native Android APP } \\
\hline
\end{tabular}

FIGURE III. FRAME STRUCTURE OF IOS AND ANDROID ON TITANIUM

Figure III takes iOS and two mobile platforms of Android as examples, describes the overall framework of the Titanium. In the Titanium framework, Web applications can easily access device UI components. For example, you can use API control navigation bar, toolbar, menu in the page provided by Titanium, and can pop-up dialog box, warning box to users dynamically. In addition, Titanium API also supports access to the local function modules, which means the user can use APIs interface provided by Titanium to access database, orientation function, file system function, network function, media function and so on.

\section{AppCan}

AppCan [9] is a cross platform mobile application development platform based on HTML5 technology, researched and developed by ZhengYi Mobile(Beijing) independent, supports cross platform mobile application development, provides mobile application management and operational control of cloud services, achieve the support and administration of the whole life cycle of mobile applications. Developers using HTML5, CSS3 and JavaScript technology can quickly develop the mobile applications that comparable to native application experience. AppCan platform provides UI rapid development framework, a local function call API interface, packaged applications system, IDE integrated development environment and local application debugging simulator, preset hundreds of sets of interface template and dozens of application plug-in to provide different application template, the interface framework is perfect, develop ment environment is humanization, resource of development is rich, strong service support, help developers get started quickly. Figure VI is the platform architecture of AppCan.

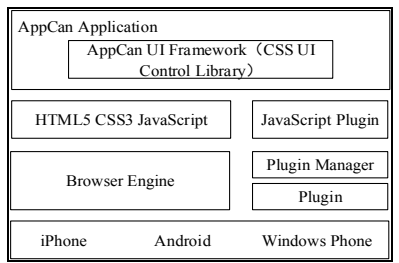

FIGURE IV. PLATFORM ARCHITECTURE OF APPCAN

\section{E. Comparison}

Although the 4 frameworks above are cross platform mobile application development framework based on HTML5, but because of their different development, their advantages and disadvantages are also different. The advantages and disadvantages of these frameworks are shown Table III.

TABLE III. CONTRAST OF THE ADVANTAGES AND DISADVANTAGES OF THE 4 FRAMEWORKS

\begin{tabular}{|c|c|c|}
\hline $\begin{array}{l}\text { framew } \\
\text { orks }\end{array}$ & advantages & disadvantages \\
\hline $\begin{array}{l}\text { PhoneG } \\
\text { ap } \\
\text { ( Cordo } \\
\text { va) }\end{array}$ & $\begin{array}{l}\text { - Support } 6 \text { platforms, } \\
\text { Compatibility is very good } \\
\text { - Support W3C } \\
\text { standardization technology } \\
\text { - Rich plug-in } \\
\text { - Open source and free } \\
\text { - Low development costs }\end{array}$ & $\begin{array}{l}\text {-The quality of the } \\
\text { third party plug-in is } \\
\text { uneven } \\
\text { - Slow running speed }\end{array}$ \\
\hline $\begin{array}{l}\text { Sencha } \\
\text { Touch }\end{array}$ & $\begin{array}{l}\text { - Good touch experience, } \\
\text { support a variety of gestures } \\
\text {-Customizable UI } \\
\text { - Support cloud services }\end{array}$ & $\begin{array}{l}\text { - Slow running speed } \\
\text {-Using and learning is } \\
\text { difficult } \\
\text {-High memory } \\
\text { consumption }\end{array}$ \\
\hline $\begin{array}{l}\text { Titaniu } \\
\mathrm{m}\end{array}$ & $\begin{array}{l}\text { - Open source and free } \\
\text { - Rapid prototype } \\
\text { development } \\
\text { - Design based on web } \\
\text { - Security }\end{array}$ & $\begin{array}{l}\text { - The base component } \\
\text { is free, but the } \\
\text { advanced components } \\
\text { and the official } \\
\text { guidance is charge } \\
\text { - Error prompt is not } \\
\text { accurate }\end{array}$ \\
\hline AppCan & $\begin{array}{l}\text { - Integrated solutions, simple } \\
\text { environmental installation } \\
\text { - Provide UI } \\
\text { - Rich API interface } \\
\text { - Support for local package } \\
\text { and remote package }\end{array}$ & $\begin{array}{l}\text { - Not open source, the } \\
\text { underlying code can't } \\
\text { be optimized } \\
\text { - Free version need to } \\
\text { upload the source code, } \\
\text { security is not } \\
\text { guaranteed } \\
\text { - Installation package } \\
\text { is large }\end{array}$ \\
\hline
\end{tabular}

IV. AdVANTAGES AND Disadvantages OF CROSS PlatForm DEVELOPMENT MODE BASED ON HTML5

\section{A. Advantages}

Compared to the traditional native development model, cross platform development model based on HTML5 has following advantages: 
1)Cross platform: Compared to the traditional native applications. Hybrid app can easily across the platform adaptation, and support the mainstream mobile intelligent terminal operating system, such as Android and IOS. At the same time, hybrid app can solve a variety of types of display screen compatibility issues, can be universally compatible different display device.

2)Low development costs: HTML, CSS and JavaScript and other commonly used web language is mainly used in the development of the front end of hybrid app. As more personnel are engaged in web development, so labor cost relatively is low. On the other hand, web language learning difficulty curve is less than the native application development language, and reduces the incubation time of the developer.

3)Update and maintenance is convenient: Hybrid app can be very convenient to achieve the application of automatic updates, needn't to uploaded install package to the application store.

\section{B. Disadvantages}

The lack of cross platform development model based on HTML5 can be attributed to two aspects: one is the shortage of HTML5 itself, and the other is the shortage of development framework.

1)The shortage of HTML5: Although HTML5 standard specification has completed and released, but the HTML5 also has some shortcomings, the first one is the security issues. WebStorage, WebSocket has serious security problems, can easily be exploited by hackers to steal user information and data. Secondly, HTML5 is a recommended standard, rather than mandatory standards, so many Internet companies, such as Google, Firefox and opera, because of their own interests, in the HTML5 support is not complete, resulting in the performance of HTML5 browser is not good, thus user experience is not good.

2)The shortage of development framework: HTML, JavaScript, CSS are running on the WebKit which is a part of operating system, subject to the impact of WebKit processing speed. Compared to native applications, HTML5 application running slow, performance is poor. Secondly, platform of cross platform framework based on HTML5 depends on the third party framework itself, resource scheduling rate is less than $100 \%$.

\section{SUMMARY AND OUTLOOK}

Cross platform mobile hybrid application development model based on HTML5 can be very convenient to achieve cross platform adaptation, can reduce the cost of development and maintenance. Different frameworks have their own advantages and disadvantages, a reasonable use of mobile applications can make the development of more simple, also allows users experience better.
With the further development of HTML technology, it is believed that HTML5 can go farther in the direction of cross platform, can also adapt to the future development needs of mobile applications, builds better, more secure application. With browser kernel and mobile terminal performance continuing to raise, HTML5 hybrid applications will run more smoothly on the mobile terminal, more close to the operating speed of native applications. With the improvement and development of HTML5 cross platform development framework, hybrid application development will become more popular, the cost of development will be greatly reduced. With these bottlenecks being resolved, cross platform mobile hybrid application development based on HTML5 will also become more dominant.

\section{REFERENCES}

[1] Gartner. Gartner Says Worldwide Smartphone Sales to Slow in 2016, (2016). Available online at: http://www.gartner.com/newsroom/id/3339019/.

[2] Gartner. Gartner Says Worldwide Smartphone Sales Grew 3.9 Percent in First Quarter of 2016, (2016). Available online at:http://www.gartner.com/newsroom/id/3323017/.

[3] Bouras C, Papazois A, Stasinos N. A Framework for Cross-platform Mobile Web Applications Using HTML5[C]//Future Internet of Things and Cloud (FiCloud), 2014 International Conference on. IEEE, 2014: 420-424.

[4] Wilken J. Ionic in Action: Hybrid Mobile Apps with Ionic and AngularJS[M]. MANNING PUBN, 2015. pp. 1-7.

[5] Adobe PhoneGap. Build amazing mobile apps powered by open web tech. Available online at: http://www.phonegap.com/.

[6] Apache Cordova. Available online at: http://cordova.apache.org/

[7] Sencha Touch 2.4. Available online at: http://docs.sencha.com/touch/2.4/index.html

[8] Appcelerator. Available online at: http://docs.appcelerator.com/platform/latest/\#!/guide

[9] AppCan.Available online at: http://www.appcan.cn/ 